\title{
Desenvolvimento de uma escala de mensuração de características de gamificação para usuários de aplicativos em dispositivos móveis
}

\author{
Richard D'arc da Silva Brito, Luis Hernan Contreras Pinochet ${ }^{1}$, \\ Evandro Luiz Lopes e Mauri Aparecido de Oliveira \\ Universidade Federal de São Paulo - UNIFESP, São Paulo, SP, Brasil
}

DETALHES DO ARTIGO
Histórico do Artigo:
Recebido: 15 de Março de 2017
Aceito: 13 de Outubro de 2017
Disponível online: 27 de Dezembro de 2017
Sistema de revisão "Double blind review"
Editor Científico
Leonardo da Silva

Palavras-chaves:

Gamificação;

Desenvolvimento de escala;

Modelagem de equações estruturais.

\begin{abstract}
RESUMO
O objetivo deste artigo é apresentar o desenvolvimento de uma escala de mensuração de características de gamificação para usuários de aplicativos em dispositivos móveis. O modelo desenvolvido foi inspirado no framework denominado Octalysis criado por Yu-kai Chou. Definida uma versão preliminar da escala foi necessária a realização do procedimento de validação de face conduzida com 12 juízes. Após essa fase inicial, encadeou-se a purificação da escala, via cálculo da confiabilidade, e a aplicação do modelo a partir de um pré-teste conduzido com 30 respondentes. A próxima etapa foi aplicar um survey que contou com a participação de 452 respondentes de todo o país. A abordagem de pesquisa quantitativa obedeceu uma fase descritiva exploratória e outra subsidiada pela aplicação da Modelagem de Equações Estruturais. Como resultado, os juízes confirmaram 6 dos 8 construtos propostos de modelo original, e por meio das análises realizadas junto com a amostra de usuários de aplicativos em dispositivos móveis, foi possível verificar que estes 6 construtos confirmados confirmaram a significância estatística da escala desenvolvida. Logo, o modelo proposto neste estudo é consistente, podendo ser aplicado em futuras pesquisas.
\end{abstract}

(c) 2018 Internext | ESPM. Todos os direitos reservados!

\section{INTRODUÇÃO}

Os jogos sempre foram um passatempo comum para a humanidade e, com os constantes avanços da era digital e a disseminação da internet, não era difícil imaginar que os jogos eletrônicos prevalecessem e se expandissem (Terlutter \& Capella, 2013).

Em 2011 as pessoas já passavam em média 3 bilhões de horas por semana jogando, e esse número só vem aumentando. porque um jogo é uma oportunidade para os indivíduos concentrarem sua energia em algo em que são bons e distrair-se da ânsia por um trabalho mais satisfatório, para um sentido mais forte de comunidade, e para uma vida mais significativa (McGonigal, 2011).

Novas tecnologias vêm sendo criadas para inspirar a motivação das pessoas e auxiliá-las no

\footnotetext{
${ }^{1}$ Contato do autor - Email: luis.hernan@unifesp.br
}

desenvolvimento de comportamentos benéficos, sejam eles individuais ou coletivos. A tendência mais popular nessa área é a gamificação, ou gamification, no termo original, que se refere ao uso de tecnologias empenhadas em promover motivações intrínsecas, por meio do emprego de diversas características encontradas em jogos (Kim, 2015).

A literatura científica, nos últimos cinco anos, apresentou poucos modelos que já foram aplicados para encontrar explicações sobre os efeitos da gamificação. Jorge e Sutton (2016) desenvolveram um modelo de gamificação, denominado Funication, que possibilita às organizações transformarem seus ambientes internos em ambientes gamificados. Os demais modelos encontrados na literatura servem como norteadores de aplicações práticas da 
gamificação, tais como o Gamified ID Model, criado para orientar o projeto e desenvolvimento de um curso gamificado (Asleitner, 2000; Becker, 2015).

Assim, devido à escassez de modelos que propõem a mensuração das características da gamificação, este trabalho busca contribuir com a construção de um modelo original baseado no framework desenvolvido por Chou (2014), testandoo empiricamente. Desta forma, esta pesquisa propõe o estudo das técnicas de gamificação que vêm sendo utilizadas em aplicativos para dispositivos móveis pelos seus usuários, e como objetivo, desenvolver uma escala de características de gamificação para usuários de aplicativos em dispositivos móveis.

A consultoria Gartner Group apresentou em seu artigo "The use of game mechanics and experience design to digitally engage and motivate people to achieve their goals", que a Gamification vai além do simples entretenimento tradicional dos jogos, e utiliza de seus elementos para inspirar a motivação das pessoas e engajá-las em diferentes objetivos, tais como: aprendizado, desenvolvimento e resolução de problemas (Lee \& Hammer, 2011; Burke, 2015). Além disso, o relatório Gamification Market da consultoria Solution (2016) reforça a estimativa de crescimento do mercado de gamificação de US\$ 1,65 bilhão em 2015 para US\$11,10 bilhões em 2020.

Pesquisadores têm argumentado que o uso de aplicativos móveis é uma evolução na forma de aplicativos de serviços das mídias sociais, principalmente, em função da integração entre diferentes aplicações (Hong et al., 2013; Oghuma et al., 2016). Isto sugere que o uso em aplicativos em dispositívos móveis (smartphone ou tablet) não é apenas um aspecto utilitário orientado para tarefas específicas, mas que pode despertar aos usuários uma reflexão maior no processo de comunicação. $\mathrm{Na}$ verdade, uma forte demanda por gamificação já está presente nestes aplicativos móveis. Conforme pesquisa de Andriotis (2014), 79\% dos jovens consideram ser mais produtivos se as atividades realizadas na universidade ou no trabalho fossem mais semelhantes a um jogo gamificado em aplicativo em dispositivo móvel.

Dessa maneira, este artigo procura apresentar o tema da gamificação, uma vez que devido à poucas publicações científicas a respeito dessa temática, o conceito é pouco difundido entre as escolas de gestão, tornando-se restrito aos cursos voltados à tecnologia. Pela mesma razão, poucos modelos foram aplicados para encontrar explicações sobre os efeitos da gamificação. Nesta perspectiva é que nasce a oportunidade em propor um protocolo de construção de escalas de mensuração de características de gamificação para usuários de aplicativos em dispositivos móveis.

O artigo está assim estruturado: inicialmente são apresentados os fundamentos teóricos identificados na literatura; os procedimentos metodológicos; a análise dos dados; e, finalmente as conclusões.

\section{REFERENCIAL TEÓRICO}

Nesta seção serão apresentados os conceitos teóricos e estudos que fundamentaram esta pesquisa. Com o objetivo de facilitar o entendimento do tema abordado, o referencial teórico foi subdividido em dois assuntos principais: o contexto geral dos jogos e da gamificação; e as características da gamificação.

\subsection{0 contexto geral dos jogos e da gamificação}

Os jogos têm sido usados para exercitar a mente, entreter crianças e adultos, e para integrar pessoas socialmente há milhares de anos (Terlutter \& Capella, 2013). Com a expansão dos jogos eletrônicos e a popularização dos smartphones, a importância e o papel dos jogos na sociedade cresceu rapidamente. A recente propagação de games para áreas além do entretenimento, como marketing e educação, mostrou ser um ponto chave para a realidade econômica e social (Jagoda, 2014).

Os jogos são sistemas estruturados e desafiadores, que tornam o processo de aprendizado gratificante, permitindo profundo engajamento, senso de autonomia e que pede aos usuários, que sejam os heróis de suas próprias histórias. Ao deixar de lado as diferenças encontradas em cada gênero de jogo e suas complexidades tecnológicas, todos os jogos compartilham quatro características que os definem: metas, regras, sistema de feedback e participação voluntária (Salen \& Zimmerman, 2003; McGonigal, 2011; Vianna et al., 2013; Chang \& Wei, 2016).

A gamificação ou gamification no termo original, que se refere ao uso de tecnologias empenhadas em promover motivações intrínsecas, por meio do emprego de diversas características dos jogos em outros domínios, fora da indústria do entretenimento, como na educação, administração 
pública, marketing, política e saúde, é uma tendência emergente, que deriva da gigantesca popularidade dos jogos e de suas intrínsecas capacidades de motivar a ação, seja para resolução de problemas ou potencializar a aprendizagem em diversas áreas do conhecimento e da vida das pessoas (Hunter, 2012; Schell, 2014; Kim, 2015).

Portanto, a gamificação emprega diversas características encontradas em jogos. Desse modo, é comum a dificuldade de diferenciar um jogo de um serviço ou aplicativo que utiliza a gamificação, não obstante, é importante ressaltar que criar uma solução gamificada não implica no desenvolvimento de um jogo (Fardo, 2013). Assim, a gamificação utiliza parte das características definidoras de jogos, de modo que o resultado final não seja um jogo completo.

\subsection{As Características da gamificação}

A gamificação utiliza diversos elementos oriundos de jogos para estimular a motivação, tais como pontuação, placar de líderes, emblemas ("badges" no original), moedas virtuais, narrativa e avatares (Dicheva et al., 2015). Entre os autores que estudam a gamificação é comum a utilização de estruturas, ou frameworks de elementos que são criados com o objetivo de tornar o processo mais claro e organizado. É uma forma de estudar a aplicação da gamificação em produtos já lançados e nortear sua utilização em novos produtos e projetos.

O framework escolhido para nortear este estudo é denominado Octalysis (Figura 1). Publicada originalmente no ano de 2014, essa estrutura é baseada em um octógono com oito pilares da gamificação, ou drivers no original, representando cada um dos lados. Este framework é de autoria de Yu-kai Chou - palestrante internacional sobre gamificação. O autor vem desenvolvendo pesquisas empresariais, inclusive em organizações inovativas tais como: Google, Stanford University e TEDx (TEDxLausanne realizado em 2014). Em 2015, o autor foi classificado como o \#1 entre os Gamification Gurus Power 100 da RISE, e também foi premiado

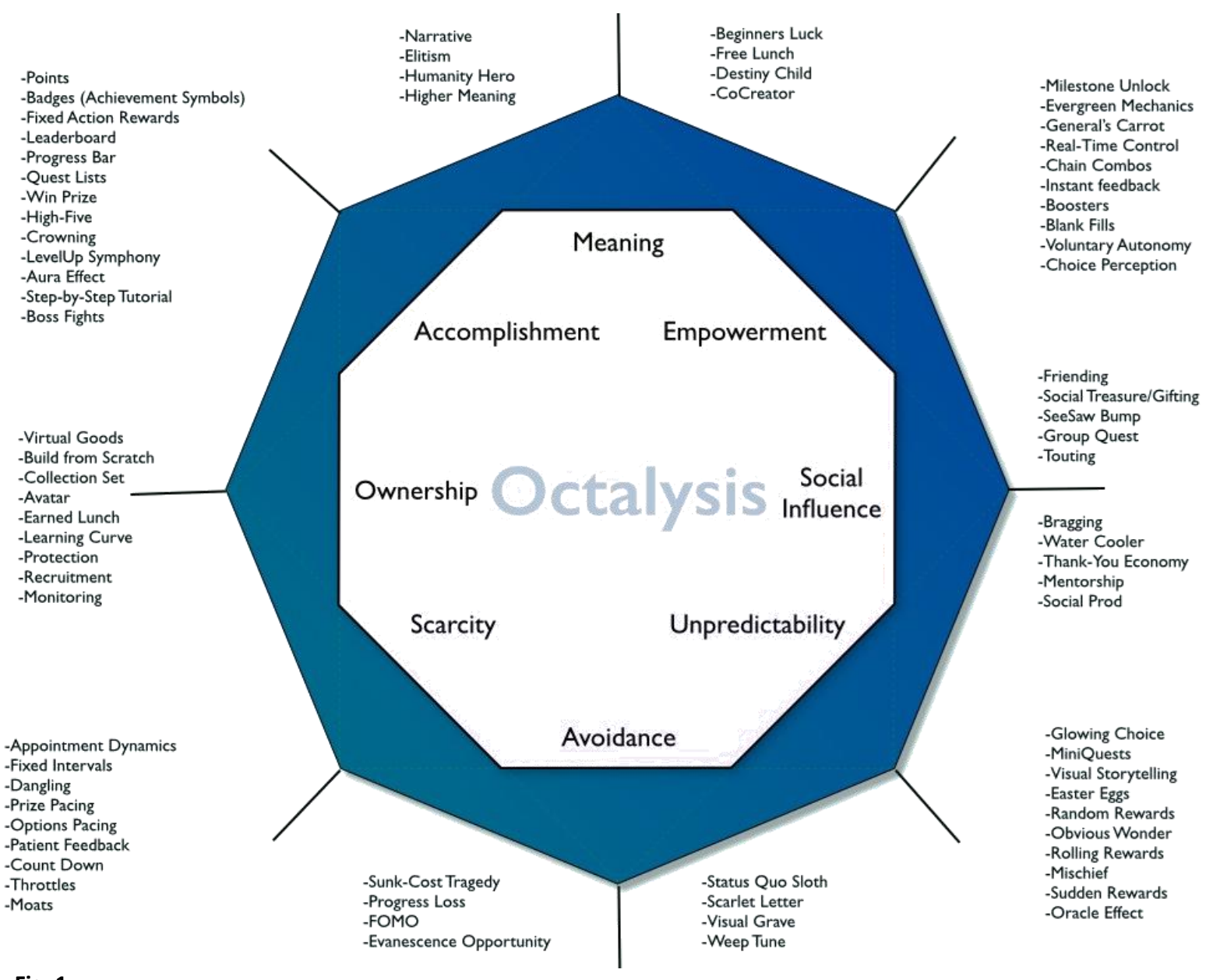

Fig. 1

Octalysis Framework (Chou, 2014). 
com o Gamification Guru of the Year Award em 2014 e 2015 pelo Congresso Mundial de Gamificação baseado na Europa.

O primeiro driver é denominado Epic Meaning \& Calling (Significado Épico e o Chamado). Este pilar está associado com a implementação do objetivo significativo no qual as pessoas possam acreditar que estão contribuindo para algo maior do que elas mesmas, ou que foram escolhidas para fazer alguma coisa. O principal conceito deste pilar é unir o objetivo dos usuários à característica altruísta da natureza humana (Kanov et al., 2004). Ao combinar esses dois elementos cria-se um caminho comportamental para o altruísmo que é a recompensa aos usuários por ações que beneficiam a comunidade como um todo (Zichermann \& Cunningham, 2011).

Development and Accomplishment (Desenvolvimento e Realização) é o segundo driver e relaciona-se com a sensação de progresso, desenvolvimento de habilidades e a conquista de façanhas complexas seguidos por uma recompensa ou sensação de grande realização. Esse é o pilar mais comumente utilizado em projetos de gamificação, normalmente são usadas técnicas de pontuação, emblemas, barras de progresso e placares de líderes. O cérebro humano tem um desejo natural de sentir o progresso, crescimento e de ver os números subindo (Sailer et al., 2013). Nos games, os jogadores normalmente começam em níveis extremamente simples e progressivamente se movem para os complexos, criando um sistema que visa encorajar a continuidade do jogo (Medler, 2011). Indicadores de desempenho fornecem objetivos adicionais aos jogadores, provocando competição amigável e comparação entre usuários (Montola et al., 2009).

O terceiro pilar, Empowerment of Creativity (Fortalecimento da Criatividade), é utilizado para envolver os usuários em um processo criativo que podem expressar sua individualidade, precisam descobrir formas diferentes de abordar um desafio e tentar reinventar o sistema à sua vontade. As pessoas querem e precisam de oportunidades para expressar sua autonomia e originalidade. Os indivíduos querem possibilidades de distinguir-se dos demais ao seu redor. Esse sentimento está diretamente ligado ao desejo humano de mostrar um senso de estilo, identidade e personalidade e mostrar uma identificação com um grupo ou comunidade (Kapp, 2012; Zichermann \& Cunningham, 2011).
Ownership and Possession (Propriedade e Possessão) é o quarto pilar, associado a motivação das pessoas que estão diretamente relacionadas com os chamados "bens virtuais" ou "moedas virtuais", em que a utilização e comercialização tornou-se extremamente popular e um modelo importante de receitas para serviços on-line, redes sociais, ou jogos multijogador massivo on-line (Hamari \& Lehdonvirta, 2010). Lehdonvirta, Wilska e Johansson (2009) examinaram a propriedade de itens virtuais por uma perspectiva sociológica, e por meio de um estudo de caso, descobriram que os bens virtuais são, normalmente utilizados para sinalizar distinções entre pessoas.

Social Influence (Influência Social) é o quinto pilar do modelo Octalysis e está relacionado com atividades inspiradas pelo que as pessoas pensam, fazem ou dizem umas sobre as outras, e inclui todos os elementos sociais que as motivam: aceitação, competição, inveja, necessidade de não se sentir excluído e companheirismo. Uma importante fonte de conhecimento sobre si mesmo vem da comparação com os outros (Wood, 1989; Gilbert et al., 1995; Nan, 2008; Suls et al., 2002). Nos aplicativos gamificados os usuários ganham pontos e são classificados com base no número total de pontos acumulados, e é comum a exibição do desempenho dos outros (Vorderer et al., 2003).

O sexto pilar denominado Scarcity \& Impatience (Escassez e Impaciência) tem por conceito chave a motivação da inocapacidade em obter algo imediatamente porque há uma grande dificuldade em obtê-la, pois, esse driver trabalha com a tendência natural de querer algo que não se pode ter. As técnicas que envolvem escassez podem ser classificadas em dois tipos: limite de quantidade e limite de tempo. Limite de quantidade é uma técnica em que o indivíduo é informado que o produto, serviço ou ação não pode ser garantido dado a um estoque limitado (Cialdini, 1985).

Unpredictability \& Curiosity (Imprevisibilidade e Curiosidade) é o sétimo pilar que contempla as técnicas que flertam com a vontade dos usuários em descobrir o que irá acontecer (Zichermann \& Cunningham, 2011). A gamificação utiliza curiosidade por meio de ações que podem oferecer recompensas surpresa ou o estímulo gerado nas pessoas ao saber que podem ganhar algo a qualquer momento enquanto jogam (Hidi et al., 2004; Marczewski, 2016). 
O oitavo e último driver da estrutura Octalysis, Loss \& Avoidance (Perda e Evitação), é baseado na teoria de aversão à perda, em que os indivíduos são mais motivados a agir se estão correndo risco de perder algo em comparação com a motivação de ganhar algo (Kahneman \& Tversky, 1979). Em outras palavras, se há uma pequena chance das pessoas perderem algo que ganharam, elas farão o possível para evitar a perda (Ariely et al., 2005).

\section{PROCEDIMENTOS METODOLÓGICOS}

Este tópico apresenta o design do estudo, contendo: o desenvolvimento da escala, o sujeito de pesquisa, seus instrumentos, procedimentos e tratamento dos dados e os modelos propostos.

\subsection{Desenvolvimento da escala, conceituação e geração de itens}

O protocolo proposto foi construído com o objetivo de apresentar os passos lógicos para a construção de uma escala de mensuração de características de gamificação. É de ordem incremental e fruto do conhecimento e análise crítica dos principais estudos neste campo. As próximas seções apresentam a discussão suscitada sobre cada uma das etapas de um protocolo na elaboração de uma escala.

A elaboração de escalas de mensuração envolve a construção de um instrumento em si e a associação de conceitos qualitativos com as métricas quantitativas, ou seja, a atribuição de números a objetos segundo alguma regra determinada (Pooja \& Sagar, 2012), a qual busca disciplinar o estudo do fenômeno. Com tal direcionamento, um protocolo de elaboração de escalas é um conjunto organizado de etapas a cumprir, com o uso adequado de técnicas selecionadas, para se construir uma escala de mensuração válida (Rossiter, 2002).

A elaboração de qualquer escala inicia-se com a definição de seus construtos, dado que, sem a definição correta do que será medido, qualquer mensuração será imprecisa. Nesse sentido, considera-se um construto como sendo um termo conceitual utilizado para descrever teoricamente um fenômeno de interesse (Edwards \& Bagozzi, 2000).

Inicialmente foi realizada uma pesquisa aprofundada da literatura disponível em bases de dados científicas, permitindo uma visualização clara do tema em termos de pesquisas acadêmicas e a identificação dos melhores modelos para avaliar as dimensões propostas. Assim, os construtos deste estudo foram baseados no modelo Octalysis, e como proposto por Chou (2014) foram utilizados oito construtos para avaliar as dimensões propostas: Propósito e Chamado para ação; Desenvolvimento e Realização; Fortalecimento da criatividade; Propriedade; Influência Social; Escassez e Impaciência; Imprevisibilidade e Curiosidade; Perda e Evitação.

A geração de itens para essa nova escala foi baseada nesse modelo, a partir do qual foram gerados inicialmente 74 itens distribuídos nas oito dimensões de análise previamente determinadas, seguindo como critério fundamental a adequação semântica aos construtos, e que mantivessem com estes uma relação claramente refletiva, viabilizando a correta mensuração da escala (Jarvis, Mackenzie \& Podsakoff, 2003). Após definida uma versão preliminar da escala, fez-se necessário a realização de uma validação de face (DeVellis, 2003). A validação de face foi aprofundada por meio dos juízes (Malhotra, 2014).

Conforme sugerido por Hardesty e Bearden (2004) esta etapa teve o objetivo de observar a concordância do grupo convidado de especialistas, aqui denominados juízes, com a capacidade da escala de mensurar cada construto pretendido e se os itens da escala estão claros e adequados para a estimação, bem como os subsídios para se efetuar o cálculo da confiabilidade da escala em uma segunda etapa (Gountas et al., 2012).

O uso de duas fases para a etapa da purificação é indicado por estudos como de Gountas et al. (2012), pois as etapas são complementares e a validação de face confere a vantagem de se ter um instrumento mais propenso à aprovação pelos testes estatísticos.

O procedimento de validação de face incluiu, inicialmente, o convite a juízes (especialistas) para a realização de retorno em questionário encaminhado por e-mail. Durante a realização da validação de face, na tentativa de obter maior objetividade, os juízes foram convidados a preencher três formulários (Face Validity Motivação Positiva, Face Validity Motivação Neutra e Face Validity Motivação Negativa), no qual assinalaram um número em cada um dos itens, relacionando os construtos com suas respectivas assertivas que estavam dispostas aleatoriamente (DeVellis, 2003; Hardesty \& Bearden, 2004). A avaliação da capacidade de relacionar a variável com o construto conferiu uma pontuação. Valores de 0,65 
ou maiores foram considerados aceitáveis como níveis de concordância convergente (Stratman \& Roth, 2002) ou acima de 0,80 conforme Hair et al. (2006).

O objetivo desta etapa, como afirmam DeVellis (2003) e Bright et al. (2012), é verificar, em um grupo de especialistas, se os itens da escala podem ser considerados adequados à mensuração de um construto. Após a definição da validação de face, encadeia-se, pela elaboração do protocolo, a purificação da escala via cálculo da confiabilidade no qual foi realizada a aplicação do modelo via questionário eletrônico para uma amostra de respondentes. No caso deste trabalho, tal fase foi subsidiada pela aplicação da Modelagem de Equações Estruturais (MEE).

Para a composição do painel de juízes, foram convidados por conveniência 20 profissionais produtivos acadêmica e cientificamente, buscando indivíduos que estejam dentro do perfil desejado ao estudo, isto é, pesquisadores e ou profissionais envolvidos diretamente com a temática da gamificação. Todos os profissionais contatados aceitaram participar do estudo, no entanto, 8 deles não deram retorno à pesquisa, deixando de enviar suas respostas.

O instrumento, elaborado foi enviado aos vinte peritos para análise de conteúdo de seus itens, com relação a: forma de apresentação do instrumento; facilidade de leitura; compreensão e clareza dos itens; e pertinência conceitual (abrangência do conteúdo) referente a gamificação.

Os juízes foram convidados a participar do estudo mediante contato realizado pelo pesquisador, via email, em que foram expostos: o título, os objetivos do estudo e a forma de avaliação do instrumento, solicitando a colaboração dos mesmos dentro do prazo de quinze dias. Foram encaminhados 12 pareceres dentro do prazo estipulado. Após o recebimento dos pareceres de cada juiz (experts na área), realizou-se uma análise descritiva das respostas acerca dos itens do instrumento proposto e, a partir dessa análise, foram realizados os ajustes sugeridos. Todos os juízes são pesquisadores que possuem publicações em congressos e periódicos científicos de referência na área, ou pertencem a grupos de pesquisa que têm aderência com o tema da gamificação.
Em síntese, os peritos concordaram que o instrumento proposto contempla as características da gamificação, e que os itens abrangem os aspectos que permeiam os construtos propostos. Este procedimento gerou algumas correções de enunciado para facilitar o entendimento, indicações de itens pouco relevantes, além da exclusão de dois construtos: "Fortalecimento da criatividade" e "Escassez e Impaciência". Após a validação de face, todos os itens foram reavaliados com base nas sugestões e críticas obtidas nos resultados dos questionários pelos juízes, ficando 33 itens subdivididos entre as 6 principais dimensões pesquisadas, sendo 4 assertivas para "Propósito e Chamado para ação"; 7 para "Desenvolvimento e Realização"; 6 para "Propriedade"; 6 para "Influência Social"; 6 para "Imprevisibilidade e Curiosidade"; e 4 para "Perda e Evitação".

\subsection{Participantes, instrumento de coleta, procedimentos, tratamento de dados e validade}

A população analisada foi constituída de usuários de aplicativos móveis em smartphones em maior ou menor grau. Os dados foram coletados por conveniência, resultando em técnica de amostragem não probabilística, limitando, portanto, generalização dos resultados desta pesquisa (Kim \& Malhotra, 2005).

O presente estudo foi desenvolvido tendo como base dados coletados por meio de uma survey, adaptado para testar o modelo de escala desenvolvida pelo autor, e descrita nos tópicos anteriores. Esse instrumento de coleta de dados foi composto por quarenta e seis assertivas que foram respondidas por meio de uma escala de Likert, com end points ancorados em 1=discordo totalmente e $5=$ concordo totalmente. Há ainda, questões demográficas. Os questionários foram preenchidos, pelos próprios respondentes, na presença dos pesquisadores, após uma breve apresentação da pesquisa.

O instrumento de coleta de dados foi elaborado em formato eletrônico com o objetivo de apresentar ao participante os itens de mensuração identificados na fase anterior do estudo. Já a coleta de dados foi composta por meio da técnica de questionário eletrônico, que foi auto aplicado via internet, por meio da plataforma TypeForm a partir de um link, podendo ser respondido utilizando dispositivos móveis ou desktops. O link de acesso foi 
disponibilizado pelo Facebook e em fóruns de discussão on-line, tais como: o Fórum UOL e o Fórum Outerspace que foram escolhidos de forma intencional, por suas discussões, em sua maioria, serem em torno de jogos e tecnologia. Os dados foram coletados entre os meses de agosto a outubro de 2016.

Hair et al. (2006) ressalta que o tamanho da amostra para o pré-teste deve ter no mínimo quatro e no máximo trinta indivíduos. Por outro lado, Malhotra (2014) afirma que o tamanho de amostra do pré-teste deve variar entre quinze e trinta entrevistados. Desta maneira, o modelo foi submetido a um pré-teste conduzido em 30 sujeitos da pesquisa para verificar se existiu compreensão do instrumento de pesquisa. A próxima etapa foi aplicar a survey com a ampliação da amostra e foram obtidos ao total 452 questionários, não houve necessidade de exclusão de questionários por preenchimento incorreto ou campos em branco.

Para esta pesquisa, optou-se pelo modelos baseados em mínimos quadrados parciais, ou abreviadamente, PLS (Partial Least Squares). Devido à constatação da presença de distribuição não normal nos conjuntos de dados de diversas variáveis

Tab 1

Detalhamento dos Construtos

\begin{tabular}{|c|c|c|c|}
\hline Construto $^{1}$ & Octalysis $^{2}$ & Descrição & Principais referências \\
\hline Propósito & $\begin{array}{l}\text { Epic Meaning } \\
\quad \& \text { Calling }\end{array}$ & $\begin{array}{l}\text { Este construto está associado com a motivação que as pessoas } \\
\text { têm ao acreditar que estão contribuindo com um propósito } \\
\text { maior, ou que foram escolhidas para fazer alguma coisa. }\end{array}$ & $\begin{array}{l}\text { Kanov et al. (2004) } \\
\text { Zichermann e } \\
\text { Cunningham (2011) }\end{array}$ \\
\hline Desenvolvimento & $\begin{array}{l}\text { Development \& } \\
\text { Accomplishment }\end{array}$ & $\begin{array}{l}\text { Este construto relaciona-se com a sensação de progresso, } \\
\text { desenvolvimento de habilidades e a conquista de façanhas } \\
\text { complexas seguidos por uma recompensa ou sensação de } \\
\text { grande realização. }\end{array}$ & $\begin{array}{l}\text { Montola et al. (2009) } \\
\text { Medler (2011) } \\
\text { Sailer et al. (2013) }\end{array}$ \\
\hline Propriedade & $\begin{array}{l}\text { Ownership } \\
\text { \& Possession }\end{array}$ & $\begin{array}{l}\text { Construto associado à necessidade das pessoas por sentirem } \\
\text { que possuem algo dentro do aplicativo ou algum aspecto dele. } \\
\text { Tem por base o princípio de que ao possuirmos alguma coisa, } \\
\text { sentimos a necessidade de melhorá-la, protegê-la e obtermos } \\
\text { o máximo de sua capacidade. }\end{array}$ & $\begin{array}{l}\text { Lehdonvirta et al. (2009) } \\
\text { Hamari e Lehdonvirta } \\
\text { (2010) }\end{array}$ \\
\hline Influência Social & Social Influence & $\begin{array}{l}\text { Este construto está relacionado com atividades inspiradas pelo } \\
\text { que as pessoas pensam, fazem ou dizem umas sobre as outras. }\end{array}$ & $\begin{array}{l}\text { Wood (1989) } \\
\text { Gilbert et al. (1995) } \\
\text { Suls et al. (2002) } \\
\text { Vorderer et al. (2003) } \\
\text { Nan (2008) }\end{array}$ \\
\hline Imprevisibilidade & $\begin{array}{l}\text { Unpredictability } \\
\quad \& \text { Curiosity }\end{array}$ & $\begin{array}{l}\text { Este construto está associado com a curiosidade e o } \\
\text { inesperado. Utiliza a vontade que os indivíduos tem de } \\
\text { descobrir o que irá acontecer. }\end{array}$ & $\begin{array}{l}\text { Hidi et al. (2004) } \\
\text { Zichermann e } \\
\text { Cunningham (2011) } \\
\text { Marczewski (2016) }\end{array}$ \\
\hline Evitação & $\begin{array}{c}\text { Loss \& } \\
\text { Avoidance }\end{array}$ & $\begin{array}{l}\text { Este construto relaciona-se com o medo de perder algo ou } \\
\text { desejo de evitar que um evento negativo ocorra. Diretamente } \\
\text { ligado à teoria da aversão à perda. }\end{array}$ & $\begin{array}{l}\text { Kahneman e Tversky } \\
\text { (1979) } \\
\text { Ariely et al. (2005) }\end{array}$ \\
\hline
\end{tabular}

Observações: ${ }^{1}$ Modelo proposto adaptado de Chou (2014); ${ }^{2}$ Nomenclatura de Chou (2014).

Fonte: Elaboração própria da amostra, optou-se pela utilização do approach Partial Least Squares Structural Equation Modeling (PLS-SEM), levando então ao uso do aplicativo SmartPLS Professional v.3.

\subsection{Modelo de validação das características da gamificação}

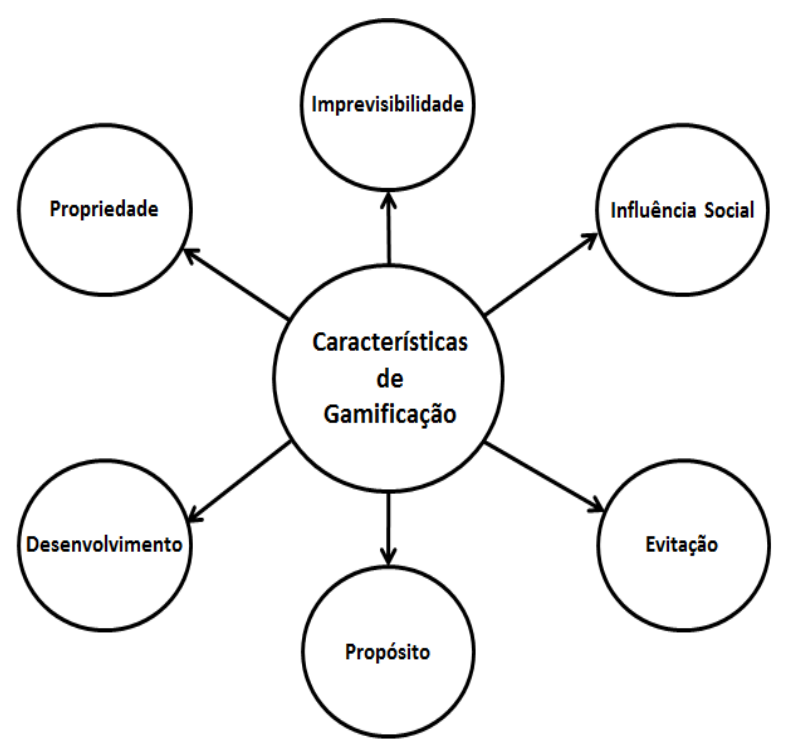

Fig. 2

Modelo final de validação das características da gamificação Fonte: Elaboração própria. 
O modelo de pesquisa proposto nesta pesquisa (ver Figura 2) foi desenvolvido com base no Framework Octalysis criado por Chou (2014) no qual subdivide as características da gamificação em oito diferentes construtos apresentados anteriormente e que foram adaptados conforme a validação de face realizada.

A Tabela 1 detalha os construtos que estão presentes na composição final do modelo de validação das características da gamificação proposto neste estudo.

\section{ANÁLISE DOS RESULTADOS}

Nesta seção são apresentadas as análises, descritiva e estimativa do modelo estrutural em relação as variáveis latentes e suas implicações nos resultados.

\subsection{Caracterização dos respondentes}

As características dos respondentes desta pesquisa são aqui apresentadas para possibilitar a contextualização da realidade socioeconômica dos participantes deste estudo. Na Tabela 2 estão detalhadas as informações sobre gênero, faixa etária, região do país, nível educacional e renda familiar.

Analisando os dados demográficos, verifica-se que, a maior parte dos respondentes são do gênero masculino correspondendo a $63,05 \%$ da amostra. Dos respondentes $80,53 \%$ tem idade entre 16 e 30 anos. É possível observar que embora os respondentes sejam de todas as regiões do país, a maioria $(71,68 \%)$ residem na região sudeste do Brasil, 60,84\% estão cursando graduação e $23,67 \%$ possui renda familiar de $R \$ 4.427,36$ a $R \$ 8.695,88$.

Ambos os gêneros apresentam equilíbrio dentro de cada divisão socioeconômica, ficando em sua maioria entre $\mathrm{R} \$ 2.409,01$ a $\mathrm{R} \$$ 8.695,88 (público masculino $45,1 \%$ e feminino $46,4 \%$ ) , apenas na faixa entre de $\mathrm{R} \$ \mathbf{8 . 6 9 5 , 8 8}$ a $\mathrm{R} \$ 20.272,56$ o gênero masculino apresenta maior número. Além disso, as regiões Norte e Nordeste apresentam semelhante divisão socioeconômica, ficando em sua maioria de $R \$ 4.427,36$ a $R \$ 8.695,88$. A região Centro-oeste apresentou maior percentual de respondentes na faixa de renda de $\mathrm{R} \$ 639,78$ a $\mathrm{R} \$ 1.446,24$. Por outro lado, a região Sul possui uma concentração maior de entrevistados na faixa entre $\mathrm{R} \$ 4.427,36$ a $\mathrm{R} \$$ $20.272,56$.
Tab. 2

Caracterização dos respondentes

\begin{tabular}{|c|c|c|c|}
\hline Característica & Tipo & $\mathrm{N}$ & $\begin{array}{c}\text { Freq. } \\
(\%)\end{array}$ \\
\hline \multirow[t]{3}{*}{ Gênero } & Masculino & 285 & 63,05 \\
\hline & Feminino & 167 & 36,95 \\
\hline & Total & 452 & 100 \\
\hline \multirow[t]{11}{*}{ Faixa etária } & De 16 a 20 & 86 & 19,03 \\
\hline & De 21 a 25 & 195 & 43,14 \\
\hline & De 26 a 30 & 83 & 18,36 \\
\hline & De 31 a 35 & 45 & 9,96 \\
\hline & De 36 a 40 & 21 & 4,65 \\
\hline & De 41 a 45 & 8 & 1,77 \\
\hline & De 46 a 50 & 2 & 0,44 \\
\hline & De 51 a 55 & 5 & 1,11 \\
\hline & De 66 a 70 & 2 & 0,44 \\
\hline & Missing & 5 & 1,11 \\
\hline & Total & 452 & 100 \\
\hline Região & Região Norte & 19 & 4,20 \\
\hline \multirow[t]{6}{*}{ do País } & Região Nordeste & 29 & 6,42 \\
\hline & Região Centro-Oeste & 31 & 6,86 \\
\hline & Região Sudeste & 324 & 71,68 \\
\hline & Região Sul & 46 & 10,18 \\
\hline & Missing & 3 & 0,66 \\
\hline & Total & 452 & 100 \\
\hline \multirow[t]{8}{*}{ Educacional } & Nível Médio Incompleto & 2 & 0,44 \\
\hline & Nível Médio Concluído & 23 & 5,09 \\
\hline & Cursando graduação & 275 & 60,84 \\
\hline & Graduação concluída & 81 & 17,92 \\
\hline & Cursando pós-graduação & 24 & 5,31 \\
\hline & Pós-graduação concluída & 44 & 9,73 \\
\hline & Missing & 3 & 0,66 \\
\hline & Total & 452 & 100 \\
\hline \multirow{9}{*}{$\begin{array}{l}\text { Renda } \\
\text { familiar }\end{array}$} & Menor $\mathrm{R} \$ 639,78$ & 5 & 1,11 \\
\hline & de $R \$ 639,78$ a $R \$ 1.446,24$ & 30 & 6,64 \\
\hline & de $R \$ 1.446,24$ a $R \$ 2.409,01$ & 48 & 10,62 \\
\hline & de $R \$ 2.409,01$ a $R \$ 4.427,36$ & 99 & 21,90 \\
\hline & de $R \$ 4.427,36$ a $R \$ 8.695,88$ & 107 & 23,67 \\
\hline & de $R \$ 8.695,88$ a $R \$ 20.272,56$ & 71 & 15,71 \\
\hline & Maior que $\mathrm{R} \$ 20.272,56$ & 17 & 3,76 \\
\hline & Missing & 75 & 16,59 \\
\hline & Total & 452 & 100 \\
\hline
\end{tabular}

Fonte: elaboração própria.

\subsection{Premissas para o uso da modelagem de equações estruturais}

Nas técnicas de análise multivariada que utilizam variáveis métricas e testes estatísticos, a normalidade multivariada é a condição fundamental de aplicação. A normalidade dos dados foi verificada por meio de observação da curtose e assimetria presente na amostra de dados pelo teste Kolmogorov-Smirnov e o respectivo p-value de cada variável. Este procedimento foi necessário para limitar a possibilidade da utilização de algumas técnicas de análise estatística que tenham como característica a distribuição normal dos dados. Em relação as variáveis preditoras relacionadas com a variável 
latente "gamificação" no contexto do tema desta pesquisa foi possível acomodar a multicolinearidade no modelo (todos os valores dos Fatores de Inflação de Variância (VIFs) ficaram abaixo de 5, pois o VIF menor foi 1,083 e o maior 2,144). Todos os $p$-value do teste referente aos indicadores resultaram significativos com $p<0,01$. Ademais, o resultado do teste de normalidade, por não encontrar aderência dos dados à distribuição gaussiana corroborou com a estimação do modelo estrutural ao analisar a correlação das variáveis em mínimos quadrados parciais (Hair et al., 2014; Ringle et al., 2014).

\subsection{Análise do modelo das características de gamificação}

A análise do modelo de mensuração deve preceder a análise das relações entre as variáveis latentes. Durante a primeira iteração, para a análise da validade convergente foram apresentados os resultados das cargas fatoriais obtidas por cada uma das variáveis latentes, observou-se que duas variáveis possuíam valores inferiores a 0,5 (Ringle et al., 2014). Neste caso, foi necessário eliminar as variáveis PROP01 $(0,362)$ e DES06 $(0,454)$ o que possibilitou a adequação do modelo. O próximo passo foi examinar as Variâncias das Médias Extraídas (AVE) e as correlações quadráticas entre os construtos, a validade convergente, e a confiabilidade composta.

Após os ajustes inicias do modelo verificou-se que em alguns construtos das AVEs estavam com valores abaixo de 0,5. Segundo Ringle et al. (2014) nestas situações deve-se eliminar variáveis observadas ou mensuradas dos construtos que apresentam carga fatorial baixa. Para se elevar o valor da AVE devem-se eliminar as variáveis com cargas fatoriais de menor valor. Neste caso, foi necessário eliminar as variáveis: DESO5=0,629; $\quad$ EVITO2=0,655; $\quad$ IMPO4=0,538; ISO2=0,584; $\quad$ PRO03=0,348; $\quad$ PRO05=0,572; e finalmente, DESO2=0,659 para aumentar o valor da AVE.
Nesse sentido, foi possível a adequação do modelo e todas as variáveis latentes apresentaram variância média extraída maior que 50\%, o que atinge os critérios para a indicação da existência de validade convergente chegando na escala de mensuração proposta (Apêndice 1).

Dado que todas as variáveis de um questionário utilizam a mesma escala de medição, o coeficiente é calculado a partir da variância dos itens individuais (Tabela 3). Os alphas de Cronbach variaram entre 0,549 e 0,758. Sendo que valores acima de 0,60 a 0,75 são considerados moderados, e de 0,75 a 0,90 altos. A confiabilidade composta consiste na avaliação realizada a partir dos resultados obtidos do modelo de análise fatorial confirmatória para os coeficientes de mensuração e dos erros de medida. Assim, as confiabilidades compostas variaram entre 0,763 e 0,838, o que é considerado "muito bom". Para este modelo as AVEs variaram entre 0,511 e 0,581 . Além disso, $R^{2}$ value mensura a acurácia preditiva do modelo, representando os efeitos combinados das variáveis endógenas sobre as variáveis exógenas. No presente estudo, o $R^{2}$ value demonstrou que o modelo possui acurácia e relevância preditiva em todos os construtos, o que representa, acurácia preditiva substancial analisando as variáveis apresentadas (Malhotra, 2014).

A validade discriminante avalia se os itens que refletem o fator não apresentam correlação excessiva com outros fatores. Pelo critério de Fornell e Larcker (1981), a validade discriminante fica demonstrada quando as variâncias médias extraídas forem superiores ou iguais ao quadrado da correlação entre os fatores ou, de outro modo, quando a raiz quadrada das variâncias médias do contruto for maior que a correlação do construto com as demais variáveis latentes do modelo analisado. Na Tabela 4 observam-se que todas as variâncias extraídas médias são superiores ou igual ao quadrado da correlação entre os fatores, assim, não

Tab. 3

Análise das relações entre os construtos

\begin{tabular}{lccccc}
\hline \multicolumn{1}{c}{ Construtos } & $\begin{array}{c}\text { No } \\
\text { Itens }\end{array}$ & $\begin{array}{c}\text { Cronbach's Alpha } \\
(>0,7)\end{array}$ & $\begin{array}{c}\text { Composite Reliability } \\
(>0,7)\end{array}$ & $\begin{array}{c}\text { (AVE) } \\
(>0,5)\end{array}$ & $\mathrm{R}^{\mathbf{2}}$ \\
\hline Desenvolvimento & 4 & 0,723 & 0,828 & 0,546 & 0,629 \\
Evitação & 3 & 0,549 & 0,763 & 0,520 & 0,409 \\
Imprevisibilidade & 4 & 0,737 & 0,835 & 0,560 & 0,676 \\
Influência Social & 5 & 0,758 & 0,838 & 0,511 & 0,486 \\
Propriedade & 4 & 0,716 & 0,825 & 0,541 & 0,607 \\
Propósito & 3 & 0,629 & 0,804 & 0,581 & 0,582 \\
\hline
\end{tabular}

Fonte: Elaboração própria. 
foi necessária a eliminação de itens do modelo de mensuração.

Tab. 4

Validade Discriminante: Critério de Fornell-Larcker

\begin{tabular}{lcccccc}
\hline \multicolumn{1}{c}{ Variáveis latentes } & $(1)$ & $(2)$ & (3) & (4) & (5) & (6) \\
\hline (1)Desenvolvimento & 0,739 & & & & & \\
(2) Evitação & 0,457 & 0,721 & & & & \\
(3) Imprevisibilidade & 0,571 & 0,381 & 0,748 & & & \\
(4) Influência Social & 0,317 & 0,411 & 0,487 & 0,715 & & \\
(5) Propriedade & 0,722 & 0,498 & 0,565 & 0,334 & 0,736 & \\
(6) Propósito & 0,511 & 0,335 & 0,607 & 0,515 & 0,480 & 0,762 \\
\hline
\end{tabular}

Nota: A diagonal em destaque apresenta as raízes quadradas da AVE Fonte: Elaboração própria.

Após as fases de validação convergente e discriminante, passou-se à analise dos caminhos estruturais. O resultado dessa fase é apresentado na Figura 3.

O modelo proposto nesta pesquisa foi estimado utilizando-se a técnica bootstrapping, comparando a amostra original com as amostras geradas por essa técnica. Nesse sentido, foram geradas outras 500 amostras e realizado o teste $t$ Student conforme é apresentado na Tabela 5. A análise da significância dos caminhos, de acordo com Benjamin e Gaskin (2014), pode ser verificada a partir dos $t$-values e das cargas fatoriais das variáveis observáveis. Estes valores são interpretados na sequência.

Os resultados obtidos na análise de significância dos caminhos indicaram que a maioria não obteve diferença entre a amostra original e as subamostras geradas pela técnica estatística com os limites críticos para o teste $t$ de Student, este teste permite que a análise dos coeficientes de correlação/regressão sejam iguais a zero (Hair et al., 2014). Considerou-se $t$ de Student para valores considerados significativos de $p<0,001$.

As técnicas que utilizam a vontade dos usuários descobrirem o que irá acontecer para Gamificar aplicativos em dispositivos móveis, representada pelo construto de "Imprevisibilidade" obteve, além de um coeficiente significativo, a carga mais elevada $(0,822)$, mostrando forte relação de preferência, entre os respondentes desta pesquisa, por aplicativos que continuamente fornecem informações inesperadas e imprevisíveis durante sua utilização. É interessante observar que este é um construto de gamificação negativa, isto é, possui elementos que podem deixar uma sensação desagradável no consumidor, mostrando que se aplicado

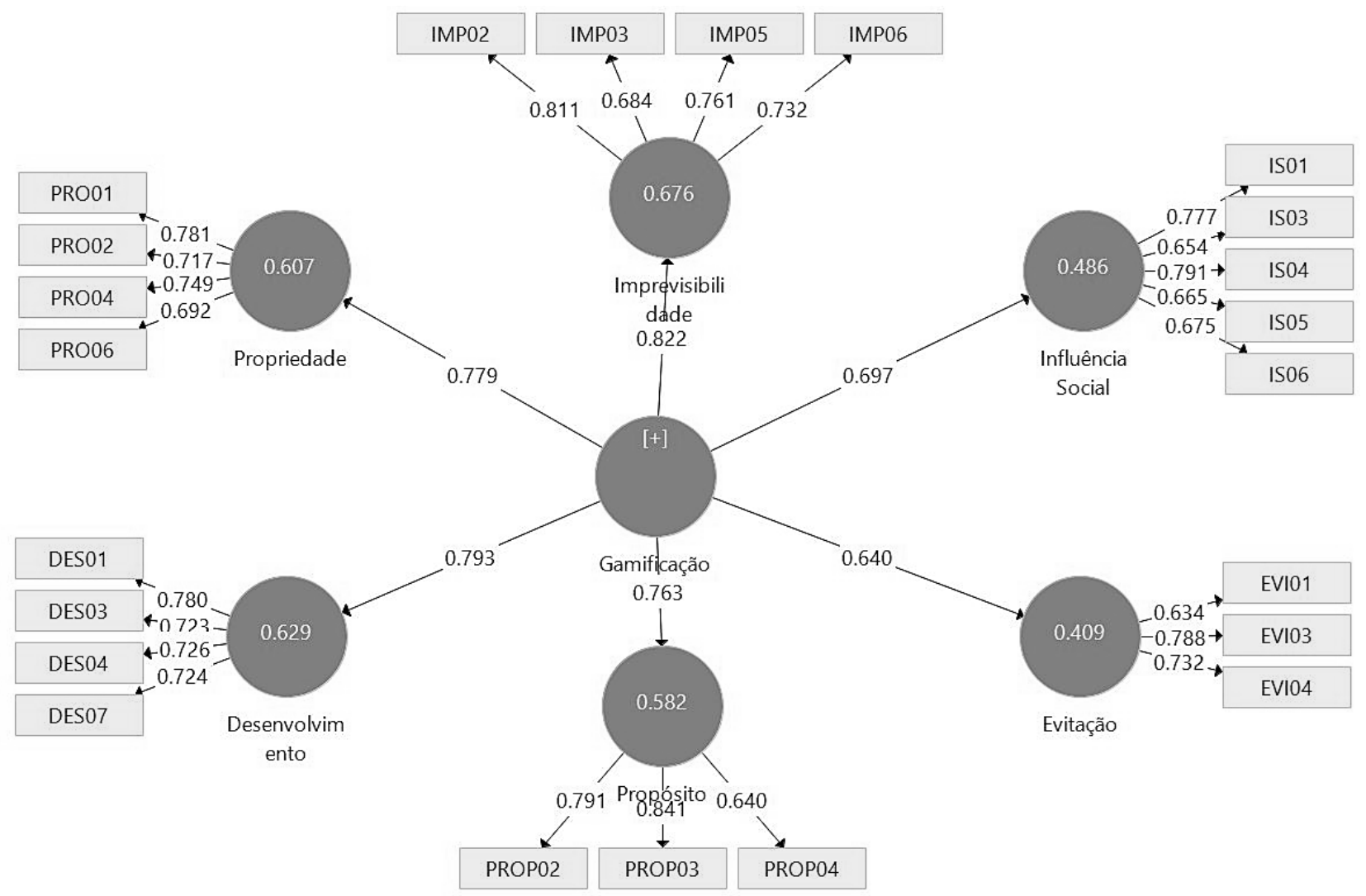

Fig. 3

Modelo de validação de características de gamificação.

Fonte: Elaboração própria. 
Tab. 5

Análise da significância dos caminhos

\begin{tabular}{lccccc}
\hline \multicolumn{1}{c}{ Path } & $(\beta)$ & Bootstrapping & SE & Teste t & P values \\
\hline Características de Gamificação -> Desenvolvimento & 0,793 & 0,795 & 0,028 & 28,214 & 0,000 \\
Características de Gamificação -> Evitação & 0,640 & 0,641 & 0,033 & 19,142 & 0,000 \\
Características de Gamificação -> Imprevisibilidade & 0,822 & 0,824 & 0,020 & 40,548 & 0,000 \\
Características de Gamificação -> Influência Social & 0,697 & 0,698 & 0,032 & 21,787 & 0,000 \\
Características de Gamificação -> Propriedade & 0,779 & 0,782 & 0,025 & 31,452 & 0,000 \\
Características de Gamificação -> Propósito & 0,763 & 0,765 & 0,026 & 28,956 & 0,000 \\
\hline
\end{tabular}

Valores críticos para $t=>1,96 p<5 \% ; 2,57 p<1 \% ; 3,64 p<0,1 \%$

Fonte: Elaboração própria.

corretamente esses elementos podem ser excelentes motivadores. Contudo, o segundo construto negativo deste estudo: "Evitação", no qual o medo de perder alguma coisa é utilizado para gamificar, segundo os entrevistados não é uma técnica tão poderosa quanto as outras e obteve o menor carga $(0,640)$.

Na sequência é possível observar a força da gamificação positiva, pois são técnicas que fazem com que o usuário perceba maior significado em suas ações ou sinta-se bem sucedido por meio de seu aprimoramento, aqui representadas pelos construtos "Desenvolvimento", que relaciona-se com a sensação de progresso e obteve a segunda carga mais elevada $(0,793)$ e "Propósito" com a carga de 0,763.

\section{CONCLUSÕES}

Este estudo é uma tentativa de aprofundar a pesquisa sobre as características de gamificação e a influência de seus elementos para usuários de dispositivos móveis, e por meio do desenvolvimento de uma escala de mensuração destas características, baseada no modelo Octalysis de Yu-kai Chou foi possível comprovar que a maioria dos construtos é aplicável dispositivos movéis e têm influência sobre o uso dos mesmos. Este fato auxilia a análise e implementação de projetos que envolvam a Gamificação no país, e consequentemente, incentiva o desenvolvimento de aplicativos móveis que utilizam tais ferramentas (Robson et al., 2014).

Ao perceber uma lacuna no que concerne ao uso não totalmente consistente de mensurações de técnicas de gamificação por escalas construídas ou adaptadas, sem os ritos próprios de elaboração e validação, desta forma, produzindo resultados num contexto sem a necessária fidedignidade metodológica, é que surge a iniciativa de se formular, com o uso de diversas técnicas, um protocolo de desenvolvimento de escalas, objetivo desta pesquisa.
Como resultado, os juízes confirmaram 6 dos 8 construtos levantados teoricamente, e por meio das análises realizadas junto com a amostra de usuários de aplicativos em dispositivos móveis, foi possível verificar que estes 6 construtos confirmados indicaram significância estatística da escala desenvolvida sobre o uso de aplicativos gamificados para dispositivos móveis. Logo, o modelo proposto neste estudo é consistente, podendo ser aplicado em futuras pesquisas.

Dos caminhos propostos pelo modelo desenvolvido nesta pesquisa, verificou-se para a amostra deste estudo, que a maiores influências sobre o uso de aplicativos gamificados são dos construtos Evitação, Imprevisiblidade e Propriedade. Portanto, os respondentes desta pesquisa são mais motivados a agir se estão correndo risco de perder algo ou se desejam evitar que isso ocorra (Kahneman \& Tversky, 1979); possuem preferências individuais por comportamentos exploratórios (Hidi et al., 2004) e sentem-se motivados ao possuirem um bem virtual que os destingue dos outros (Lehdonvirta et al., 2009).

Sugere-se em pesquisas futuras, a análise da aplicação da escala de mensuração das características da gamificação, desenvolvida neste estudo, em relação a diferentes aspectos pertinentes à gamificação, tais como: motivação, engajamento e produtividade. Da mesma forma, recomenda-se a aplicação do instrumento sobre amostras maiores e em contextos diferentes da sociedade.

As empresas poderiam ser benificiadas ao utilizarem o modelo de validação das características da gamificação, desenvolvido neste estudo para nortear a criação de produtos e serviços gamificados para aplicativos (apps). Por outro lado, i não necessariamente significa que todos os construtos devem ser utilizados simultaneamente. Assim, é evidente que cada empresa deve avaliar 
cuidadosamente seus objetivos de gamificação e as necessidades de seus clientes ou potenciais clientes, para a obtenção de melhores resultados.

De forma geral, evidenciou-se que a gamificação tem o potencial de incentivar a motivação e engajamento em diferentes contextos e ao combinar os principais elementos oriundos dos jogos, com uma proposta de valor atraente a gamificação pode ajudar as empresas a melhorarem a produtividade de seus colaboradores, por meio de metas claras e objetivos lúdicos, tornar treinamentos mais interessantes, com feedbacks constantes, competição e cooperação, ou ainda motivar seus clientes a realizar ações em aplicativos para dispositivos móveis ou websites.

Por fim, a gamificação é uma ferramenta que pode vir a possibilitar o aumento da motivação, engajamento e produtividade do envolvimento dos usuários de aplicativos gamificados. Diante disso, sugerem-se estudos que possibilitem o uso destes novos construtos (motivação, engajamento e produtivodade) como variáveis dependentes do modelo proposto.

Para as empresas é importante refletir que inserir elementos de jogos de forma não planejada, fora da realidade atual, ou da "moda", provavelmente, não irá gerar estímulos aos usuários de aplicativos. Portanto, sugere-se como recomendações gerenciais que as empresas que pretendem embarcar na jornada da gamificação busquem entender o que de fato está sendo gamificado; quais motivos; qual é o seu público (usuários ou potenciais); como será o processo de gamificação; uma estratégia adequada seria criar ou utilizar indicadores ou métricas de desempenho; testes em projetos pilotos com usuários auxiliariam que fossem desenvolvidos aplicativos gamificados com melhor rigor na qualidade; ouvir os feedbacks de usuários ou potenciais usuários poderia auxiliar na revisão sistemática nas melhorias das estratégias de gamificação; e implementação planejada de aplicativos gamificados.

\section{REFERÊNCIAS}

- $\quad$ Andriotis. N. (2014). Gamification Survey Results. Disponível em: <https://www.talentlms.com/blog/gamification-surveyresults/>. Acesso em: 20 nov. 2016.

- Ariely, D., Huber, J., \& Wertenbroch, K. (2005). When do losses loom larger than gains? Journal of Marketing Research, 42(2), 134-138.
- Asleitner, H. (2000). Designing emotionally sound instruction. Instructional Science, 28(3), 169-198.

- Becker, K. (2015). A Gamified Instructional Design Model for University Courses. Disponível em: <http://minkhollow.ca/beckerblog/2015/09/07/agamified-instructional-design-model-for-universitycourses/>. Acesso em: 14 nov. 2016.

- Burke, B. (2015). Gamification 2020: What Is the Future of Gamification? Disponível em: <https://www.gartner.com/doc/2226015/gamification-future-gamification/>. Acesso em: 21 out. 2015.

- Benjamin, P., Gaskin, J. (2014). Partial Least Squares (PLS) Structural Equation Modeling (SEM) for Building and Testing Behavioral Causal Theory: When to Choose It and How to Use It. IEEE transactions on professional communication, vol. 57, no 2 (123-146).

- $\quad$ Bright, E., Vine, S., Wilson, M. R., Masters, R. S., \& McGrath, J. S. (2012). Face validity, construct validity and training benefits of a virtual reality TURP simulator. International Journal of Surgery, 10(3), 163-166.

- $\quad$ Chang, J. W., \& Wei, H. Y. (2016). Exploring Engaging Gamification Mechanics in Massive Online Open Courses. Journal of Educational Technology \& Society, 19(2), 177-203.

- Cialdini, R. B. (2009). Influence: Science and practice. (Vol. 4). Boston, MA: Pearson Education.

- Chou, Y. (2014). Octalysis: Complete Gamification Framework. Disponível em: <http://www.yukaichou.com/gamificationexamples/octalysis-complete-gamificationframework/>. Acesso em: 22 nov. 2015.

- DeVellis, R. F. (2003). Scale development: theory and applications (2nd ed.). London: Sage Publications, Inc.

- Dicheva, D; Dichev, C; Agre, G; Angelova, G. (2015). Gamification in education: a systematic mapping study. Educational Technology \& Society, Taiwan, v. 18, n. 3, p. 75-88, 2015.

- Edwards, J. R., \& Bagozzi, R. P. (2000). On the nature and direction of relationships between constructs and measures. Psychological Methods, 5(2), 155.

- Fardo, M. L. (2013). A gamificação aplicada em ambientes de aprendizagem. Revista RENOTE - Novas Tecnologias na Educação, 11(1). ISSN 1679-1916.

- Fornell, C., Larcker, D. F. (1981). Evaluating structural equation models with unobservable variables and measurement error. Journal of Marketing Research, $v$. 18, n. 1, p. 39-50.

- $\quad$ Gilbert, D. T., Giesler, R. B., \& Morris, K. A. (1995). When comparisons arise. Journal of Personality and Social Psychology, 69 (2), 227-236.

- Gountas, J., Gountas, S., Reeves, R. A., \& Moran, L. (2012). Desire for fame: scale development and association with personal goals and aspirations. Psychology \& Marketing, 29(9), 680-689. 
- Hamari, J., \& Lehdonvirta, V. (2010). Game design as marketing: How game mechanics create demand for virtual goods. International Journal of Business Science \& Applied Management, 5(1), 14-29.

- Hardesty, D. M., \& Bearden, W. O. (2004). The use of expert judges in scale development: implications for improving face validity of measures of unobservable constructs. Journal of Business Research, 57(2), 98107.

- Hair, Jr., J., Black, W. C., Babin, B. J., Anderson, R. E. \& Tatham, R. L. (2006). Multivariate Data Analysis. 6a Ed. Upper Saddle River, NJ: Pearson Prentice Hall.

- Hair Jr., J., F.; Hult, G. T. M.; Ringle, Christian M.; Sarstedt, M. (2014). A primer partial least squares structural equation modeling (PLS-SEM). SAGE Publications, Inc.

- Hidi, S., Renninger, K.A., Krapp, A. (2004). Interest, a Motivational Variable that Combines Affective and Cognitive Functioning, In: D.Y. Dai, R.J. Sternberg (eds.), Motivation, emotion and cognition: Integrative perspectives on intellectual functioning and development, pp. 89--115, Erlbaum, Mahwah.

- Hong, J., Lee, O. K. and Shu, W. (2013). A study of the continuous usage intention of social software in the context of instant messaging, Online Information Review, Vol. 37 No. 5, pp. 692-710.

- Hunter, R. (2012). The gamification HandbookEverything you need to know about gamification. Emereo Publishing.

- Jagoda, P. (2014). Gaming the Humanities. Differences - A Journal of Feminist Cultural Studies, 25(1), 189-215.

- Jarvis, C. B.; Mackenzie S. B.; \& Podsakoff, P. M. (2003). A critical review of construct indicators and measurement model misspecification in marketing and consumer research. Journal of Consumer Research, v. 30, n. 2, p. 199-218.

- Jorge, C. F. B.; Sutton, M. J. D. (2016). Games como estratégia na construção e gestão do conhecimento no contexto da inteligência organizacional. Perspectivas em Gestão \& Conhecimento, v. 6, p. 103-118.

- Kanov, J. M., Maitlis, S., Worline, M. C., Dutton, J. E., Frost, P. J., \& Lilius, J. M. (2004). Compassion in organizational life. American Behavioral Scientist, 47(6), 808-827.

- Kahneman, D., \& Tversky, A. (1979). Prospect theory: An analysis of decision under risk. Econometrica: Journal of the econometric society, 263-291.

- Kapp, K. M. (2012). The gamification of learning and instruction: game-based methods and strategies for training and education. John Wiley \& Sons.

- Kim, B. (2015). Understanding gamification. Library Technology Reports, v. 51, n. 2.

- Kim, S. S., \& Malhotra, N. K. (2005). A Longitudinal Model of Continued IS Use: An Integrative View of Four Mechanisms Underlying Post-Adoption Phenomena. Management Science (51:5), p. 741-755.
- Lee, J. J., \& Hammer, J. (2011). Gamification in education: What, how, why bother? Academic Exchange Quarterly, 15(2), 146.

- Lehdonvirta, V., Wilska, T. A., \& Johnson, M. (2009). Virtual consumerism: case habbo hotel. Information, communication \& society, 12(7), 1059-1079.

- Malhotra, N. K. (2014). Essentials of Marketing Research: A Hands-On Orientation. Prentice Hall, 1Edition, January 20.

- Marczewski, A. (2016). Using Boredom and Curiosity to your Advantage. Disponível em: <https://www.gamified.uk/2016/06/13/usingboredom-and-curiosity-to-your-advantage/>. Acesso em: 13 nov. 2016.

- Markets and Markets. (2016). Gamification market by solution (consumer driven and enterprise driven), applications (sales and marketing), deployment type (on-premises and cloud), user type (large enterprise, smbs), industry and region - global forecast to 2020 Disponível em:

<http://www.marketsandmarkets.com/MarketReports/gamification-market-991.html/>. Acesso em: 20 nov. 2016.

- McGonigal, J. (2011). Reality is broken: why games make us better and how they can change the world. New York: The Penguin Press.

- Medler, B. (2011). Player dossiers: Analyzing gameplay data as a reward. Game Studies Journal, 11 (1).

- Montola, M., Nummenmaa, T., Lucero, A., Boberg, M., \& Korhonen, H. (2009). Applying game achievement systems to enhance user experience in a photo sharing service. In: Proceedings of the 13th International MindTrek Conference: Everyday Life in the Ubiquitous Era (pp. 94-97). ACM.

- Nan, X. (2008). The pursuit of self-regulatory goals: How counterfactual thinking influences advertising persuasiveness. Journal of Advertising, 37(1), 17-27.

- Oghuma, A. P., Libaque-Saenz, C. F., Wong, S. F., \& Chang, Y. (2016). Na expectation-confirmation model of continuance intention to use mobile instant messaging. Telematics and Informatics, Vol.33 No. 1, pp.34-47.

- Pooja, S., \& Sagar, M. (2012). High impact scales in marketing: a mathematical equation for evaluating the impact of popular scales. Advances in Management, 5(4), 31-48.

- $\quad$ Ringle, C. M.; Silva, D. da.; \& Bido, D. (2014). Modelagem de Equações Estruturais com Utilização do SMARTPLS. Revista Brasileira de Marketing - REMark. Edição Especial Vol. 13, n. 2, Maio.

- Robson, K., Plangger, K., Kietzmann, J., McCarthy, I., \& Pitt, L. (2014). Understanding gamification of consumer experiences. NA-Advances in Consumer Research volume 42.

- Rossiter, J. (2002). The C-OAR-SE procedure for scale development in marketing. International Journal of Research in Marketing, 19(4), 305-335. 
- $\quad$ Sailer, M., Hense J., Mandl, H. \& Klevers, M. (2013). Psychological perspectives on motivation through Gamification. Interaction Design and Arquitecture(s) Journal, vol. 19 pp. 28-37.

- Salen, K., \& Zimmerman, E. (2003). Rules of play: Game design fundamentals. The MIT Press.

- Schell, J. (2014). The Art of Game Design: A book of lenses. CRC Press.

- Stratman, J. K.; Roth, A. V. (2002). Enterprise Resource Planning (ERP) competence constructs: two-stage multiitem scale development and validation. Decision Sciences, v. 33, n. 4, p. 601-628.

- Suls, J., Martin, R., \& Wheeler, L. (2002). Social comparison: Why, with whom, and with what effect? Current Directions in Psychological Science, 11 (5), 159163.

- Terlutter, R., \& Capella, M. L. (2013). The gamification of advertising: analysis and research directions of in- game advertising, advergames, and advertising in social network games. Journal of Advertising , 42(2-3), 95-112.

- Vianna, Y., Vianna, M., Medina, B., \& Tanaka, S. (2013). Gamification, Inc: como reinventar empresas a partir de jogos. 1 edição, Rio de Janeiro: MJV Press.

- Vorderer, P., Hartmann, T., \& Klimmt, C. (2003, May). Explaining the enjoyment of playing video games: the role of competition. In: Proceedings of the second international conference on Entertainment computing (pp. 1-9). Carnegie Mellon University.

- Wood, J. V. (1989). Theory and research concerning social comparisons of personal attributes. Psychological Bulletin, 106 (2), 231-248.

- Zichermann, G; Cunningham, C. (2011). Gamification by Design: Implementing Game Mechanics in Web and Mobile Apps. Canada: O’Reilly Media.

\section{SOBRE OS AUTORES}

- Richard D'arc da Silva Brito é Bacharel em Administração. Pesquisador do Departamento de Administração da Escola Paulista de Economia, Política e Negócios (EPPEN) da Universidade Federal de São Paulo (UNIFESP). Seus principais temas de interesse de pesquisa é sobre Gamificação e Tecnologia.E-mail: richarddarc@outlook.com

- Luis Hernan Contreras Pinochet é Doutor em Administração. Professor do Departamento de Administração da Escola Paulista de Economia, Política e Negócios (EPPEN) da Universidade Federal de São Paulo (UNIFESP). Seus principais temas de interesse de pesquisa é sobre Tecnologia da Informação e Comunicação, e Gestão de Sistemas de Informação.E-mail: luis.hernan@unifesp.br

- Evandro Luiz Lopes é Pós-doutor em Administração. Professor do Departamento de Administração da Escola Paulista de Economia, Política e Negócios (EPPEN) da Universidade Federal de São Paulo (UNIFESP). Seus principais temas de interesse de pesquisa é sobre processo de decisão de compras e na avaliação de marcas no contexto do comportamento do consumidor. E-mail: evandro.lopes@unifesp.br

- Mauri Aparecido de Oliveira é Doutor em Administração. Professor do Departamento Multidisciplinar da Escola Paulista de Economia, Política e Negócios (EPPEN) da Universidade Federal de São Paulo (UNIFESP). Seus principais temas de interesse de pesquisa é sobre a aplicação de redes neurais artificiais na previsão de séries temporais financeiras. E-mail: mauri.oliveira@unifesp.br 


\section{APÊNDICE 1}

Escala de mensuração de características de gamificação para usuários de aplicativos em dispositivos móveis

\begin{tabular}{|c|c|c|}
\hline Construto & Item & Assertiva \\
\hline \multirow{4}{*}{ Desenvolvimento } & DES01 & Me sinto motivado a realizar ações que me dão pontos dentro de um GApp. \\
\hline & DES03 & $\begin{array}{l}\text { Me sinto motivado a continuar utilizando um GApp quando minha barra de progresso está } \\
\text { quase completa. }\end{array}$ \\
\hline & DESO4 & Utilizo com mais frequência um GApp se tenho a possibilidade de ganhar prêmios com ele. \\
\hline & DES07 & Prefiro GApps em que me sinto realizado ao vencer um desafio complexo. \\
\hline \multirow{3}{*}{ Evitação } & EVI01 & $\begin{array}{l}\text { Continuo utilizando um GApp, mesmo após ter me cansado dele, para evitar perder meu } \\
\text { progresso. }\end{array}$ \\
\hline & EVIO3 & $\begin{array}{l}\text { Sou motivado realizar rapidamente uma ação para não perder uma oportunidade única } \\
\text { dentro do GApp. }\end{array}$ \\
\hline & EVIO4 & $\begin{array}{l}\text { Me sinto motivado a continuar meu progresso quando outras pessoas podem ver que } \\
\text { estou ficando para trás no GApp. }\end{array}$ \\
\hline \multirow{4}{*}{ Imprevisibilidade } & IMP02 & Sou motivado a realizar ações que me dão recompensas surpresa no GApp. \\
\hline & IMP03 & Sou motivado a utilizar GApps que me dão uma visão diferente do ambiente ao meu redor. \\
\hline & IMP05 & $\begin{array}{l}\text { Me motiva saber que posso ganhar uma recompensa a qualquer momento enquanto } \\
\text { utilizo o GApp. }\end{array}$ \\
\hline & IMP06 & $\begin{array}{l}\text { Sou motivado a usar GApps em que posso tentar prever o que acontecerá depois ou } \\
\text { realizar palpites. }\end{array}$ \\
\hline \multirow{5}{*}{ Influência Social } & ISO1 & Prefiro GApps em que posso adicionar pessoas em uma lista de amigos. \\
\hline & ISO3 & $\begin{array}{l}\text { Prefiro GApps que me permitam mostrar ou compartilhar implicitamente minhas } \\
\text { conquistas. }\end{array}$ \\
\hline & ISO4 & $\begin{array}{l}\text { Sou motivado a usar GApps que possuem locais para compartilhar ideias e conversar com } \\
\text { outras pessoas. }\end{array}$ \\
\hline & ISO5 & Prefiro GApps em que posso orientar ou ser orientado por outras pessoas. \\
\hline & ISO6 & $\begin{array}{l}\text { Prefiro GApps que me permitem interagir de maneira simples e com poucos passos com } \\
\text { outras pessoas. }\end{array}$ \\
\hline \multirow{4}{*}{ Propriedade } & PRO01 & Prefiro GApps que me permitam colecionar recursos ou itens virtuais. \\
\hline & PRO02 & $\begin{array}{l}\text { Me sinto impelido a terminar tarefas num GApp para completar uma coleção de } \\
\text { recompensas da qual já possuo alguma parte. }\end{array}$ \\
\hline & PRO04 & Prefiro GApps em que recebo recompensas ou benefícios por conta do meu esforço. \\
\hline & PRO06 & $\begin{array}{l}\text { Prefiro GApps que me proporcionem vínculos com itens, atributos ou personagens a ponto } \\
\text { de me importar com eles. }\end{array}$ \\
\hline \multirow{3}{*}{ Propósito } & PROP02 & $\begin{array}{l}\text { Me sinto motivado a utilizar um GApp quando nele posso contribuir para um mundo } \\
\text { melhor. ("A cada acerto plantaremos uma árvore") }\end{array}$ \\
\hline & PROP03 & $\begin{array}{l}\text { Me sinto motivado a utilizar um GApp quando nele posso contribuir com um propósito } \\
\text { maior. }\end{array}$ \\
\hline & PROP04 & $\begin{array}{l}\text { Me sinto motivado a utilizar um GApp quando este me faz sentir como o único que pode } \\
\text { conquistar um desafio. ("O Escolhido") }\end{array}$ \\
\hline
\end{tabular}

Fonte: Elaboração própria.

Nota: GApp (Gamificação para Aplicativos Móveis). 


\title{
Development of a gamification characteristics measurement scale for mobile application users
}

\author{
Richard D'arc da Silva Brito, Luis Hernan Contreras Pinochet, \\ Evandro Luiz Lopes e Mauri Aparecido de Oliveira \\ Federal University of São Paulo - UNIFESP, São Paulo, SP, Brazil
}

\section{ARTICLE DETAILS}

\section{Article history:}

Received: March 15, 2017

Accepted: October 13, 2017

Available online: December 27, 2017

Double Blind Review System

\section{Scientifc Editor}

Leonardo da Silva

\section{Keywords:}

Gamification

Scale development

Structural equation modeling

\begin{abstract}
The objective of this article is to present the development of a gamification characteristics measurement scale for mobile application users. The developed model was inspired by the framework called Octalysis created by Yu-kai Chou. Defined a preliminary version of the scale, it was necessary to carry out the face validation procedure conducted with 12 judges. After this initial phase, the purification of the scale was chained, via calculation of the reliability, and the application of the model from a pre-test conducted with 30 respondents. The next step was to implement a survey that was attended by 452 respondents from all over the country. The quantitative research approach followed an exploratory descriptive phase and another subsidized by the application of Structural Equation Modeling. As a result, the judges confirmed 6 of the 8 proposed constructs of the original model, and through analyzes carried out along with the sample of mobile application users, it was possible to verify that these 6 confirmed constructs confirmed the statistical significance of the scale developed. Therefore, the model proposed in this study is consistent and can be applied in future research.
\end{abstract}

(C) 2018 Internext | ESPM. All rights reserved!

Para citar este artigo:

Brito, R. D. S.; Pinochet, L. H. C.; Lopes, E. L. e Oliveira, M. A. (2018) Desenvolvimento de uma escala de mensuração de características de gamificação para usuários de aplicativos em dispositivos móveis. Internext - Revista Eletrônica de Negócios Internacionais, 13 (1), 01-16. DOI: http://dx.doi.org/10.18568/1980-4865.1311-16

Para acessar este artigo: http://dx.doi.org/10.18568/1980-4865.1311-16 